\title{
Influencia de la morfología nanotubular en la mojabilidad y ángulo de contacto de las aleaciones Ti6Al4V ELI
}

\author{
Joan Lario $^{\mathrm{a}, \mathbb{}}$, Vicent Fombuena $^{\mathrm{a}}$, Francisco Segovia ${ }^{\mathrm{a}}$, Vicente Amigó ${ }^{\mathrm{a}}$ \\ ${ }^{a}$ Universitat Politècnica de València, Instituto de Tecnología de Materiales, Camino de Vera s/n, 46022 Valencia, España \\ ( ${ }^{\bowtie}$ Autor para la correspondencia: joalafe@posgrado.upv.es)
}

Enviado: 15 Enero 2018; Aceptado: 10 Junio 2018; Publicado on-Line: 2 Octubre 2018

\begin{abstract}
RESUMEN: La tasa de osteointegración de los implantes, entre otros factores, depende de la topografía y de la composición química de la superficie, así como de las interacciones entre la superficie del implante y las células. Con el objetivo de evaluar esta interacción, el presente trabajo evalúa la energía superficial de tres acabados superficiales a partir de la medición del ángulo de contacto. En cuanto al acabado superficial de nanotubos se ha llevado a cabo un tratamiento térmico para evaluar la influencia de este proceso en la reducción del contenido de flúor. El objetivo de este estudio es evaluar la influencia de la morfología y composición de la superficie en la mojabilidad de la aleación. La medición del ángulo de contacto se realizó empleando un goniómetro óptico. La microscopía electrónica de barrido de emisión de campo (FESEM) ha permitido realizar un estudio del diámetro y espesor de los nanotubos. El análisis de espectrometría de dispersión de energía de rayos X (EDS) se empleó para analizar la composición superficial. Como resultados, destacar que la energía superficial de los nanotubos no fotoinducidos es superior a las superficies desbastadas o grabadas con ácido. Por lo tanto, el tratamiento térmico permite reducir la ratio F/Ti en los nanotubos, reduciendo así, el ángulo de contacto e incrementando la energía superficial de los nanotubos con lo que la interacción de superficies se mejora.
\end{abstract}

PALABRAS CLAVE: Ángulo de contacto; Biomateriales; Energía superficial; Mojabilidad; Nanotubos; $\mathrm{TiO}_{2}$

Citar como/Citation: Lario, J.; Fombuena, V.; Segovia, F.; Amigó, V. (2018). "Influencia de la morfología nanotubular en la mojabilidad y ángulo de contacto de las aleaciones Ti6A14V ELI”. Rev. Metal. 54(4): e130. https://doi.org/10.3989/ revmetalm. 130

\begin{abstract}
Influence of the nanotubular morphology on the wettability and contact angle of Ti6 Al4V ELI alloy. The implant Osseointegration rate depends, among other factors, on the surface's topography and chemical composition, as in the interactions between the implant surface and cells. In this work the surface free energy for three surface treatments was calculated through contact angle measurements. For the anodic oxidized samples, a heat treatment was carried out to evaluate the influence of this process on the reduction of fluorine content. The aim of this work was study the influence of surface morphology and chemical composition on the alloy's wettability behavior. The contact angle measurement was analyzed by the sessile drop method. The nanotubes morphology was evaluated by Field Emission Scanning Electron Microscopy (FESEM). The surface composition was analyzed by Energy Dispersive Spectroscopy (EDS). The non-photoinduced nanotubes surface free energy was higher than the polished or acid etched samples. The heat treatment lowered the F/Ti ratio in the nanotube and decreased the contact angle, increasing the interaction with the surface.
\end{abstract}

KEYWORDS: Biomaterials; Contact angle; Nanotubes; Surface free energy; $\mathrm{TiO}_{2}$; Wettability

ORCID: Joan Lario (https://orcid.org/0000-0003-4843-3334); Vicent Fombuena (https://orcid.org/0000-0001-7266-6205); Francisco Segovia (https://orcid.org/0000-0002-4143-5751); Vicente Amigó (https://orcid.org/0000-0002-2107-0273)

Copyright: (C) 2018 CSIC. Este es un artículo de acceso abierto distribuido bajo los términos de la licencia de uso y distribución Creative Commons Reconocimiento 4.0 Internacional (CC BY 4.0). 


\section{INTRODUCCIÓN}

Las prótesis metálicas deben presentar una biocompatibilidad que permita retrasar las revisiones quirúrgicas, bien por degradación de las prótesis o bien por posibles efectos citotóxicos que causen una degradación de la salud del paciente. La mejora, a corto plazo, de la biocompatibilidad se centra en el desarrollo de tratamientos superficiales a escala nanométrica que aumenten la vida útil de los implantes (Lario et al., 2016a). La modificación de la capa superficial de óxido de titanio puede proporcionar un medio más adecuado para la absorción de proteínas y el crecimiento celular, acelerando el proceso de regeneración del hueso y mejorando a corto y largo plazo la osteointegración de los implantes (Kim et al., 2013; Lee et al., 2013).

La osteointegración es un proceso que implica la adhesión, diferenciación y proliferación de los osteoblastos (Das et al., 2008). Entre los diferentes factores que influyen en la biocompatibilidad de los implantes, la composición de la superficie, resistencia química, rugosidad, mojabilidad y energía superficial son unos de los parámetros más importantes que pueden influir en las interacciones entre las células y la aleación de titanio. Además de las características superficiales del implante, la osteointegración también depende otros factores como son la calidad del hueso receptor (Duraccio et al., 2015), del diseño del implante (Szmukler-Moncler et al., 1998) y las solicitaciones mecánicas al que está sometido (Niinomi, 2008).

Entre los tratamientos superficiales empleados comercialmente para modificar la rugosidad se pueden citar el arenado y el tratamiento ácido. La combinación de ambos tratamientos superficiales ha demostrado mejorar la osteointegración al compararlo con los implantes mecanizados o que únicamente presentaban uno de los dos tratamientos (Herrero-Climent, 2013). Dentro de los tratamientos superficiales se encuentran aquellas técnicas que permiten producir una capa de óxido. Los resultados del estudio de Elias et al. (2008) muestran como los implantes anodizados presentan un torque para separar el implante del hueso un $46 \%$ superior que los implantes mecanizados, indicando que ha existido un mayor crecimiento óseo en las primeras etapas de osteointegración. La oxidación electroquímica, con electrolitos que contienen iones de flúor, está centrando el interés de los investigadores dado que permite obtener una estructura nanotubular de óxido de titanio (Kubota et al., 2004; Minagar et al., 2013; Jeong et al., 2014). La morfología y dimensiones de los nanotubos dependen del voltaje y tiempo de anodizado, de la composición y concentración del electrolito empleado, así como de la microestructura y composición de la aleación del titanio empleado (Lario et al., 2016b; Lario et al., 2017).
La mojabilidad de la superficie de un implante influye en el comportamiento celular en las primeras etapas de osteointegración. Varios estudios han demostrado que las superficies nanorugosas y nanotubulares mejoran la adhesión y crecimiento de osteoblastos, así como la segregación de colágeno y la deposición de calcio, si se compara con sus homologas con una rugosidad a escala micrométrica (Puckett et al., 2010a; Puckett et al., 2010b; Truong et al., 2010).

La mojabilidad de un líquido en una superficie se determina mediante la energía superficial, que depende de parámetros físicos, como la rugosidad, y parámetros químicos, como la composición de la superficie del sólido. Las superficies que presentan un ángulo de contacto superior a $150^{\circ}$ son normalmente clasificadas por superhidrofóbicas, mientras que las superficies que presentan un ángulo de contacto inferior a $90^{\circ}$ se clasifican como hidrofílicas. Los recubrimientos con polímeros que contienen compuestos fluorados han sido ampliamente utilizados en la industria para crear superficie superhidrofóbicas, con ángulos de contacto en agua superiores a los $150^{\circ}$ (Bharathidasan et al., 2015). Se ha confirmado que la presencia de flúor en la capa de óxido de titanio amorfa de la superficie del implante incrementa la adhesión de bacterias (Puckett et al., 2010a). Las infecciones bacterianas son uno de los principales problemas que sufren las prótesis actuales y que provocan el fallo prematuro del implante, así como evita una adecuada osteointegración entre el tejido y el implante. La formación y anclaje de un tejido óseo viable en la superficie de los biomateriales es uno de los enfoques posibles para mejorar el éxito clínico de los implantes, que se puede conseguir eliminando el contenido de flúor de los nanotubos. Sista et al. (2013) demostraron que las superficies anodizadas presentan una tasa de expresión de las proteínas fibronectina y colágeno mayor que en las superficies pulidas o grabadas con ácido. Esto aumenta el anclaje y la diferenciación de las células osteoblásticas, incrementando la biocompatibilidad del implante.

El crecimiento y diferenciación celular depende de la química de la interfase entre el hueso y el implante, que están determinados por la capa de óxido y por la composición del propio metal (Pypen et al., 1997). Posteriores tratamientos térmicos, tras el proceso de anodizado, pueden modificar su estructura cristalina de los nanotubos, eliminando el flúor retenido y mejorando con ello la biocompatibilidad (Berger et al., 2010; Zhao et al., 2010). Los resultados de Roguska et al. (2016) muestran como la actividad de los preosteoblastos MC3T3-E1 se incrementa al modificar la estructura cristalina de los nanotubos de amorfa a cristalina. La eliminación del flúor retenido en los nanotubos es aconsejable para evitar futuros procesos de degradación química, que dañen la capa de óxido de titanio y mermen la resistencia 
a corrosión del implante (Reclaru y Meyer., 1998). Varios estudios remarcan que el empleo de dentífricos que contengan compuestos fluorados acelera el proceso de degradación química de los implantes de titanio (Tomelin-Chemla et al., 1996; Fais et al., 2012; Toniollo et al., 2012).

El objetivo del trabajo ha sido obtener, mediante la técnica de anodizado electroquímico, nanotubos en la aleación Ti6A14V ELI estudiándose la influencia la morfología y un tratamiento térmico en el ángulo de contacto y energía superficial de los distintos acabados.

\section{MATERIALES Y MÉTODOS}

La aleación empleada Ti6A14V ELI ha sido suministrada por Allegheny Technologies Incorporated (ATI). Las propiedades mecánicas y composición química de la aleación, de acuerdo a los certificados del fabricante, se presentan en la Tabla 1. En esta misma tabla se incluyen las propiedades mecánicas y la composición química que debe de cumplir un Ti6A14V ELI según la norma ASTM F136 (2013).

El anodizado electroquímico, para la formación de los nanotubos, se ha realizado con una configuración convencional de dos electrodos: la aleación de titanio como electrodo de trabajo y el acero $316 \mathrm{~L}$ como contra-electrodo. Los ensayos se llevaron a cabo en un electrolito compuesto por ácido fosfórico $\left(\mathrm{H}_{3} \mathrm{PO}_{4} 1 \mathrm{M}\right)$ con adicción de un $0,8 \%$ (peso/ volumen) de fluoruro sódico $(\mathrm{NaF})$. El anodizado se realizó a temperatura ambiente durante 45 minutos, mediante DC Power Supply SM 400-AR-8 de Delta Elektronica, empleándose $15 \mathrm{~V}$ de potencial de trabajo. Después del proceso de anodizado las muestras se limpian en una solución de carbonato de sódico monohidratado $(0,25 \mathrm{M})$, durante $15 \mathrm{~min}$ con ultrasonidos, para neutralizar el electrolito. Tras la neutralización del ácido, las muestras se limpian con agua destilada y ultrasonidos durante $15 \mathrm{~min}$, y finalmente se secan con aire caliente.

El tratamiento térmico, tras el proceso de anodizado, se realiza en un horno de alto vacío $\left(<10^{-4}\right.$ mbares) Carbolite HVT $15 / 75 / 450$, a una temperatura máxima de $320{ }^{\circ} \mathrm{C}$ durante $30 \mathrm{~min}$, con una rampa de calentamiento de $5{ }^{\circ} \mathrm{C} \cdot \mathrm{min}^{-1}$. El enfriamiento se ha realizado a $10{ }^{\circ} \mathrm{C} \cdot \mathrm{min}^{-1}$ hasta temperatura ambiente.

El estudio metalográfico para identificar la microestructura, tamaño de grano, homogeneidad y distribución de los granos se ha realizado mediante microscopía óptica, con un microscopio Nikon Eclipse LV-100. Para su observación se ha realizado la preparación metalográfica de las muestras hasta estado de pulido y se ha atacado con reactivo Kroll (3 mL HF, 6ml $\mathrm{HNO}_{3}, 100 \mathrm{~mL} \mathrm{H} \mathrm{H}_{2} \mathrm{O}$ ) para revelar su microestructura. La microscopía electrónica de barrido de emisión de campo (FESEM ULTRA 55 de Zeiss), equipada con un detector de EDS de Oxford Instruments Ltd., se ha empleado para estudiar la morfología y composición de los nanotubos. Con el programa abierto de tratamiento de imagen ImageJ, ha sido posible medir el diámetro y espesor de los nanotubos a partir de las imágenes obtenidas en FESEM tras el tratamiento de anodizado electroquímico y también tras el tratamiento térmico.

El estudio de los ángulos de contacto, formados por los cuatro líquidos patrón de diferente naturaleza y polaridad, permite realizar un cálculo de la energía superficial del sustrato de Ti6A14V ELI con diferentes acabados superficiales. El equipo empleado para la medición del ángulo de contacto fue el goniómetro óptico, modelo "Easydrop Standadrd FM140" del fabricante KRÜSS. Los valores de ángulo de contacto fueron obtenidos del análisis geométrico de las imágenes de las gotas, empleando el software comercial "Drop Shape Analysis SW21". Como mínimo se tomaron 10 medidas en cada uno de los acabados superficiales y de estas mediciones se obtuvo el valor promedio. Los líquidos patrón utilizados en este estudio fueron el agua purificada, diiodometano (99\% pureza) suministrado por Acros Organics (Acros Organics, Geel, Belgica) y la formamida (grado ACS) y glicerol ( $99 \%$ pureza) suministrados por Scharlau Chemie S.A (Scharlab S.L., Barcelona, España). Los ángulos de contacto de los cuatro líquidos empleados aparecen recopilados en la Tabla 2. El método de cálculo utilizado para calcular la energía superficial ha sido el de OwensWendt, Ec. (1) (Owens y Wendt, 1969). Este método

TABLA 1. Propiedades mecánicas y composición química del Ti6Al4V ELI

\begin{tabular}{|c|c|c|c|c|c|c|c|c|c|c|}
\hline & \multicolumn{3}{|c|}{ Propiedades mecánicas } & \multicolumn{7}{|c|}{$\begin{array}{c}\text { Composición química } \\
(\% \text { peso })\end{array}$} \\
\hline & $\begin{array}{l}\text { Resistencia a la } \\
\text { tracción (MPa) }\end{array}$ & $\begin{array}{l}\text { Límite } \\
\text { elástico } \\
(\mathrm{MPa})\end{array}$ & $\underset{(\%)}{\text { Alargamiento }}$ & Al & $\mathbf{V}$ & $\mathbf{O}$ & $\mathrm{Fe}$ & $\mathbf{C}$ & $\mathbf{N}$ & $\mathbf{H}$ \\
\hline $\begin{array}{l}\text { AlEACIÓN DE } \\
\text { ESTUDIO }\end{array}$ & 1124 & 1020 & 21 & 6,0 & 4,0 & 0,12 & 0,18 & 0,03 & 0,01 & $>0,01$ \\
\hline $\begin{array}{l}\text { TI6AL4V ELI } \\
\text { (UNS R56401) }\end{array}$ & $830-896$ & $760-827$ & 15 & $\begin{array}{r}5,5 \\
-6,8\end{array}$ & $\begin{array}{r}3,5 \\
-4,5\end{array}$ & $0,13^{\mathrm{a}}$ & $0,25^{\mathrm{a}}$ & $0,08^{\mathrm{a}}$ & $0,05^{\mathrm{a}}$ & $0,01^{\mathrm{a}}$ \\
\hline
\end{tabular}

\footnotetext{
${ }^{\text {a }}$ Contenido máximo admitido.
} 
TABla 2. Características de los cuatro líquidos patrón

\begin{tabular}{lccc}
\hline Líquido & $\gamma_{L}^{d}\left(\mathbf{m} \mathbf{J} \cdot \mathbf{m}^{-2}\right)$ & $\gamma_{L}^{p}\left(\mathbf{m} \mathbf{J} \cdot \mathbf{m}^{-2}\right)$ & $\gamma_{L}\left(\mathbf{m} \mathbf{J} \cdot \mathbf{m}^{-2}\right)$ \\
\hline Agua & 22,0 & 50,2 & 72,2 \\
Glicerol & 34,0 & 30,0 & 64,0 \\
Diiodometano & 48,5 & 2,3 & 50,8 \\
Formamida & 32,3 & 26,0 & 58,3 \\
\hline
\end{tabular}

$\gamma_{L}^{d}=$ Componente Dispersivo de la Energía Libre Superficial .

$\gamma_{L}^{p}=$ Componente Polar de la Energía Libre Superficial .

$\gamma_{L}=$ Energía Libre Superficial Total.

nos permite obtener información sobre la componente polar y dispersiva de la energía superficial.

$$
\begin{gathered}
\gamma_{l} \cdot(1+\cos (\theta)) / 2\left(\gamma_{l}^{d}\right)^{1 / 2}= \\
\left(\gamma_{s}^{p}\right)^{1 / 2} \cdot\left(\left(\gamma_{l}^{p}\right)^{1 / 2} /\left(\gamma_{l}^{d}\right)^{1 / 2}\right)+ \\
\left(\gamma_{s}^{d}\right)^{1 / 2}
\end{gathered}
$$

$\gamma_{l}^{d}=$ Componente Dispersivo de la Energía Libre

Superficial del líquido

$\gamma_{l}^{p}=$ Componente Polar de la Energía Libre

$$
\text { Superficial del líquido }
$$

$\gamma_{s}^{d}=$ Componente Dispersivo de la Energía Libre

\section{Superficial de la superficial}

$\gamma_{s}^{p}=$ Componente Polar de la Energía Libre

Superficial dela superficia.

$\theta=$ Ángulo de contacto.

En esta ecuación, $\theta$ es el ángulo de contacto, $\gamma_{1}$ es la tensión superficial del líquido y $\gamma$ s es la tensión superficial del sólido o energía libre superficial. Como ya ha quedado dicho, los términos con los subíndices "d" y " $p$ " se refieren a las contribuciones dispersiva y por de cada fase. Se distingue fácilmente la forma de la ecuación matemática, del tipo " $y=a+b \cdot x "$. Así, puede representarse " $\left(\gamma_{1}^{\mathrm{p}}\right)^{1 / 2} /\left(\gamma_{1}^{\mathrm{d}}\right)^{1 / 2}$, frente a " $\gamma_{1}(1+\cos \theta) / 2\left(\gamma_{1}^{\mathrm{d}}\right)^{1 / 2}$ ". La pendiente de la recta obtenida será $\left(\gamma_{\mathrm{s}}^{\mathrm{p}}\right)^{1 / 2}$, mientras que aquel punto del eje "y" que intercepte a dicha recta será $/\left(\gamma_{1 s}{ }^{d}\right)^{1 / 2}$. La energía libre superficial total será la suma de estas dos componentes.

Todas las muestras fueron almacenadas en la oscuridad durante un periodo de dos semanas,

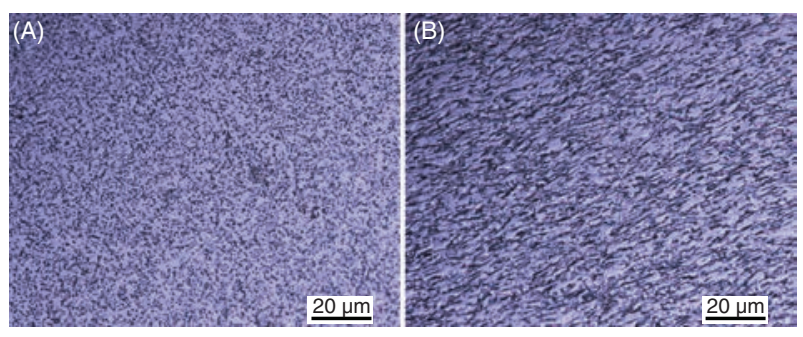

Figura 1. Metalografía de la aleación Ti6Al4V ELI 4: A) Corte transversal, y B) Corte longitudinal.

con el objetivo de evitar la superhidrofibidad fotoinducida de las superficies de óxido de titanio (Wang et al., 1997) y de esta forma simular las condiciones de almacenaje de un implante comercial.

\section{RESULTADOS}

\subsection{Análisis metalográfico}

El estudio metalográfico muestra que la aleación estudiada presenta una microestructura tipo $\alpha+\beta$, con grano no equiaxial y tamaño micrométrico o submicrométrico en función de la orientación de la muestra. La Fig. 1 presenta el corte longitudinal y transversal de la muestra tras haber sido atacada con el reactivo Kroll, donde se observa la elevada deformación que han sufrido los granos tras el proceso de cold drawn y un comienzo de recristalización provocado por un tratamiento térmico de annealing. Esta elevada deformación en frio permite reducir el tamaño de grano, endurecer la aleación e incrementar las propiedades mecánicas. Mientras que el tratamiento térmico permite aliviar tensiones residuales, incrementar la tenacidad y resistencia a fatiga de la aleación (Davis, 1990). El cambio de propiedades mecánicas se debe a la modificación de la microestructura, producida por una deformación plástica realizada a través de procesos mecánicos.

\subsection{Morfología y dimensiones de los nanotubos}

La microscopia electrónica de barrido de emisión de campo (FESEM) ha permitido observar la superficie de la aleación de titanio tras el proceso de anodizado. La Tabla 3 recopila los datos sobre el diámetro externo promedio, espesor de pared y densidad de los nanotubos obtenidos para los parámetros de anodizado empleados. En estudios anteriores realizados por los presentes autores se corroboró que el diámetro promedio de los nanotubos depende del contenido de fase $\beta$ presente en la aleación, el cual disminuye al incrementar la existencia de fase $\beta$ (Lario et al., 2016b). Además, el voltaje necesario para alcanzar el colapso de los nanotubos se incrementa con la presencia de fase $\beta$ (Lario et al., 2017). Las imágenes tomadas de las superficies de los nanotubos tras el tratamiento térmico no muestran el 
TABLA 3. Caracterización dimensional del recubrimiento de nanotubos

\begin{tabular}{|c|c|c|c|c|}
\hline Acabado superficial & $\theta_{\text {int }}(\mathbf{n m})$ & $\mathbf{E}_{\text {pared }}(\mathbf{n m})$ & $\rho_{\text {superficial }}\left(\right.$ nanotubos $\left./ \mathrm{mm}^{2}\right)$ & Longitud (nm) \\
\hline ANODIZADO 35V 45 MIN & $157 \pm 21$ & $23 \pm 3$ & $2,5 \times 10^{7}$ & 200 \\
\hline
\end{tabular}

$\theta_{\text {int }}=$ Diámetro interno de los nanotubos; $\mathrm{E}_{\text {pared }}=$ Espesor de pared; $\rho_{\text {superficial }}=$ Densidad superficial.

TABLA 4. Composición química de los diferentes acabados superficiales en las aleaciones de titanio

\begin{tabular}{lcccccc}
\hline & \multicolumn{7}{c}{ Composición química (\% peso) } \\
\cline { 2 - 7 } Acabado superficial & $\mathbf{T i}$ & $\mathbf{A l}$ & $\mathbf{V}$ & $\mathbf{O}$ & $\mathbf{F}$ & $\mathbf{N a}$ \\
\hline DESBASTADO & $89,7 \pm 0,4$ & $6,6 \pm 0,2$ & $3,7 \pm 0,2$ & 0,0 & 0,0 & 0,0 \\
GRABADO ÁCIDO & $90,9 \pm 0,1$ & $5,1 \pm 0,1$ & $4,90 \pm 0,1$ & 0,0 & 0,0 & 0,0 \\
ANODIZADO & $66,4 \pm 1,4$ & $5,0 \pm 0,7$ & $2,5 \pm 0,1$ & $23,3 \pm 1,6$ & $2,4 \pm 0,5$ & $0,4 \pm 0,1$ \\
35 V 45 MIN & & & & & \\
ANODIZADO 35 V 45 MIN + & $68,8 \pm 4,7$ & $5,7 \pm 1,4$ & $2,7 \pm 0,1$ & $21,4 \pm 3,8$ & $0,5 \pm 0,2$ & $1,0 \pm 0,4$ \\
TRATAMIENTO TÉRMICO & & & & & \\
\hline
\end{tabular}

crecimiento de una capa superior que cubra a estós. Roguska et al. (2016) reportaron el crecimiento de una capa de óxido que cierra los nanotubos sobre Ti Cp tras realizar un tratamiento térmico entre $450-600{ }^{\circ} \mathrm{C}$ en aire. En nuestro caso, se ha utilizado una menor temperatura, al realizar el tratamiento térmico en alto vacío, con lo que se ha evitado la oxidación del titanio.

Uno de los inconvenientes que presentan las aleaciones de Ti6A14V ELI a la hora de obtener un recubrimiento homogéneo de nanotubos, es el fenómeno de disolución preferencial de los elementos de aleación. Además, esta disolución preferencial depende fuertemente de la microestructura, tal y como se pudo observar en estudios previos en la microestructura $\alpha+\beta$ (Lario et al., 2016b). La tasa de disolución química, en una solución donde hay presentes iones de flúor, es menor en la fase $\beta$ con respecto a las fases $\alpha$ o $\alpha^{\prime}$ (Lario et al., 2017). La longitud de los nanotubos depende de la disolución selectiva del electrolito, de ahí que mayores tiempos de anodizado obtendrán mayores longitudes de nanotubos. En este estudio fue posible obtener espesores cercanos a los $200 \mathrm{~nm}$ cuando se trabaja con tiempos de anodizado de $45 \mathrm{~min}$.

\subsection{Caracterización superficial de los tratamientos superficiales}

Mediante energías dispersivas de rayos X (EDS) se han analizado, cualitativa y cuantitativa, las superficies desbastadas, grabadas con ácido, anodizadas y anodizadas con un tratamiento térmico. La Tabla 4 recoge la composición química obtenida de estas cuatro superficies estudiadas. El análisis de la superficie de las muestras Ti6A14V ELI desbastada y grabada con ácido muestra que los únicos elementos presentes en la superficie son el titanio y los elementos de aleación. En las muestras anodizadas se observa que el pico principal es el del titanio, pero además de los elementos aleantes aparecen dos nuevos elementos (oxígeno y flúor) ausentes en las superficies mecanizadas y grabadas con ácido. La superficie anodizada está compuesta por un $66 \%$ en peso de titanio, $23 \%$ de oxígeno en forma de $\mathrm{TiO}_{2}$ y además aparece un contenido de flúor 2,4\% (Tabla 4). Tanto Macak et al. (2007) como Puckett et al. (2010a) señalan la presencia de flúor en la superficie de las aleaciones de titanio tras el proceso de anodizado electroquímico, corroborando los resultados obtenidos en este estudio.

La presencia de flúor se debe a la existencia de restos de $\left(\mathrm{TiF}_{6}\right)^{2-}$ presentes en el interior de los nanotubos, tal y como reportan otros autores (Macak et al., 2006; Berger et al., 2010). Este elemento puede afectar al crecimiento de los osteoblastos (Çalışkan et al., 2014a) y por lo tanto debe estudiarse la influencia que el tratamiento térmico puede tener en la eliminación de flúor en el interior de los nanotubos. El tratamiento térmico a $320^{\circ} \mathrm{C}$ durante $30 \mathrm{~min}$ a alto vacío permite reducir el contenido de flúor en peso del 2,4\% al 0,5\% (Tabla 4).

\subsection{Mojabilidad de los tratamientos superficiales}

El proceso de osteointegración depende de las interacciones entre las células y la superficie del implante. La topografía, composición química y energía superficial juegan un papel esencial en la adhesión celular en las prótesis. El ángulo de contacto da información sobre la mojabilidad de una superficie, siendo este un parámetro de gran influencia en el crecimiento y diferenciación de las células. La Tabla 5 muestra el ángulo de contacto para los diferentes acabados superficiales y líquidos empleados en este estudio. La Fig. 2a muestra la superficie 
TABLA 5. Ángulo de contacto aparente para los cuatro líquidos empleados en las diferentes superficies de Ti6Al4V ELI

\begin{tabular}{lcccc}
\hline Acabado superficial & Agua $\left({ }^{\circ}\right)$ & Diiodometano $\left({ }^{\circ}\right)$ & Formamida $\left({ }^{\circ}\right)$ & Glicerol $\left({ }^{\circ}\right)$ \\
\hline DESBASTADO & $72,1 \pm 9,7$ & $43,6 \pm 1,9$ & $55,4 \pm 3,3$ & $58,2 \pm 4,3$ \\
GRABADO ÁCIDO & $110,6 \pm 13,6$ & $42,4 \pm 2,1$ & $88,6 \pm 1,3$ & $93,7 \pm 1,0$ \\
ANODIZADO 35V 45MIN & $136,5 \pm 5,4$ & $37,7 \pm 5,8$ & $42,9 \pm 2,7$ & $111,0 \pm 4,6$ \\
ANODIZADO 35V 45 MIN (TT) & $134,5 \pm 1,0$ & $31,6 \pm 2,8$ & $34,7 \pm 2,3$ & $105,9 \pm 4,9$ \\
\hline
\end{tabular}

$\mathrm{TT}=$ Tratamiento Térmico

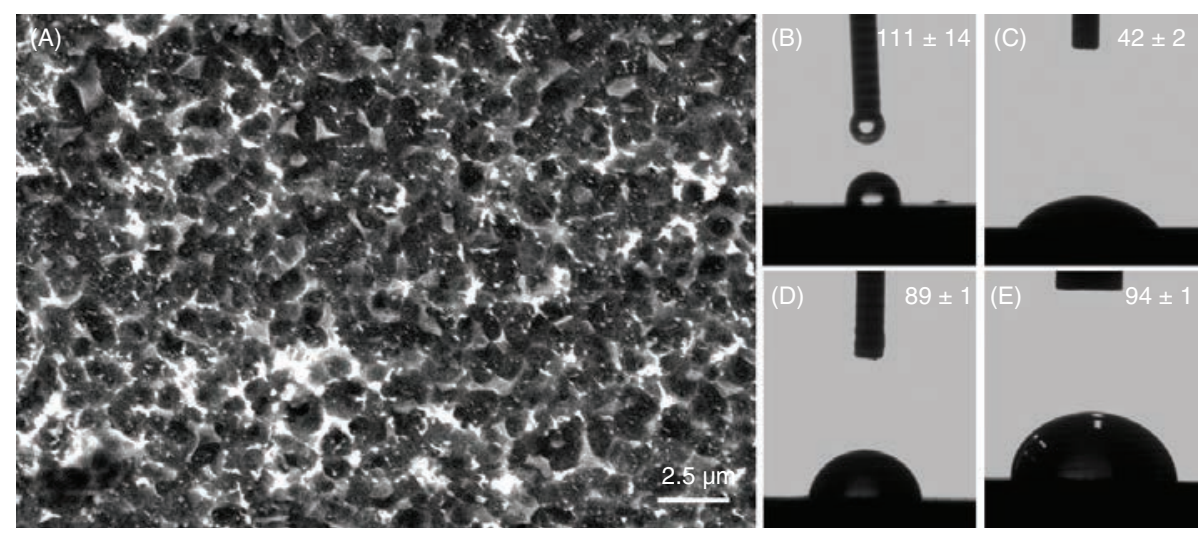

Figura 2. Mojabilidad de las superficies de Ti6A14V ELI grabada con ácido: A) Superficie Ti6Al4V ELI (SEM), B) Ángulo de contacto en agua, C) Ángulo dé contacto en diiodometano, D) Ángulo de contacto en formamida, y E) Ángulo de contacto en glicerol.

de la aleación de titanio tras haberse realizado el proceso de grabado ácido para incrementar la rugosidad.

El ángulo de contacto en agua se incrementa significativamente al incrementar la rugosidad de la superficie, pasándose de valores cercanos a los $111^{\circ}$ para una microrugosidad alcanzada con el grabado ácido a valores promedios de $136^{\circ}$ para una rugosidad a escala nanométrica obtenida mediante el anodizado electroquímico (Tabla 5). El mayor grado de hidrofobicidad de las superficies anodizadas puede estar explicado por la morfología de los nanotubos. $\mathrm{Al}$ trabajar con $35 \mathrm{~V}$ el diámetro interno de los nanotubos presenta valores próximos a los $160 \mathrm{~nm}$ (Tabla 3), incrementando la probabilidad de que el aire pueda quedar atrapado en el interior de los nanotubos y por debajo de la gota del líquido, alcanzándose el estado de Cassie-Baxter (Giacomello et al., 2012).

Para poder estudiar la influencia que presentan los átomos de flúor retenidos en la energía superficial se ha realizado el tratamiento térmico en las muestras anodizadas a $35 \mathrm{~V}$ durante 45 minutos (Fig. 3). La presencia del flúor incrementa la hidrofobicidad de las superficies de $\mathrm{TiO}_{2}$, con morfología nanotubular. Los átomos de flúor presentan una baja polarizabilidad y una alta electronegatividad $(3,98)$, comparado con el oxígeno $(3,44)$, carbono $(2,55)$ o hidrógeno $(2,20)$. Además, los enlaces titanio-flúor (Ti-F) están polarizados debido a la alta electronegatividad del flúor, lo que les otorga un momento dipolar permanente. Las superficies nanotubulares de óxido de titanio no fotoactivadas presentan el mismo comportamiento que los polímeros, es decir, superficies micro/nano texturizadas, que con la presencia de átomos de flúor generan superficies super-hidrofóbicas (Zha et al., 2017). Por lo tanto, el anodizado electroquímico sin tratamiento térmico reduce la mojabilidad de la superficie, debido a un incremento del ángulo de contacto en todos los líquidos empleados en este estudio. La cantidad de flúor presente en la superficie de los nanotubos disminuye tras el tratamiento térmico, reduciendo el ángulo de contacto e incrementando la energía superficial.

La Tabla 6 muestra la energía superficial y los valores para el componente polar y dispersivo que componen la energía superficial de las diferentes superficies, calculada de acuerdo con la Ec. (1) de Owens-Wendt. Las muestras anodizadas presentan mayor energía superficial que las muestras desbastas o grabadas con ácido. El anodizado electroquímico 

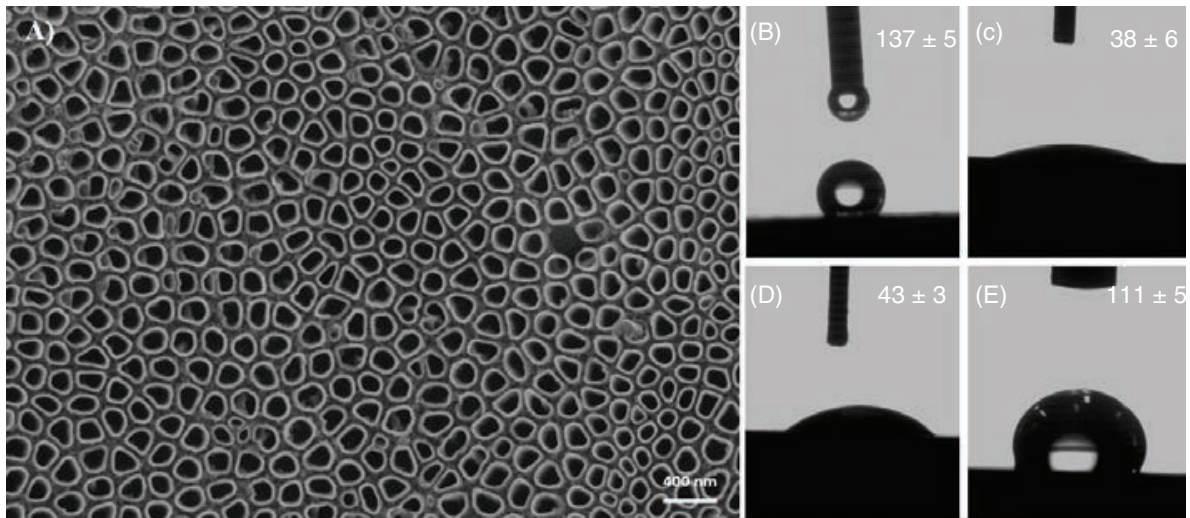

FIgura 3. Mojabilidad de las superficies de Ti6Al4V ELI anodizada $35 \mathrm{~V}$ durante $45 \mathrm{~min}$ sin tratamiento térmico: A) Superficie Ti6A14V ELI (FESEM), B) Ángulo de contacto en agua, C) Ángulo de contacto en diiodometano, D) Ángulo de contacto en formamida, y E) Angulo de contacto en glicerol.

TABLA 6. Energía libre superficial y sus componentes para los diferentes tratamientos superficiales

\begin{tabular}{lccc}
\hline & \multicolumn{3}{c}{ Energía libre superficial $\left(\mathbf{m J} \cdot \mathbf{m}^{-2}\right)$} \\
\cline { 2 - 4 } Tratamientos superficiales & Energía superficial total & Componente dispersivo & Componente polar \\
\hline DESBASTADO & 39,8 & 31,3 & 8,5 \\
GrABADO ÁCIDO & 41,9 & 40,8 & 1,1 \\
ANODIZADO 35V 45MIN. & 80,5 & 68,9 & 11,6 \\
ANODIZADO 35V 45 MIN. TT & 86,9 & 75,0 & 11,9 \\
\hline
\end{tabular}

TT $=$ Tratamiento Térmico

en una solución que contiene iones de flúor genera una morfología a escala nanométrica que aumenta la energía superficial total. Se ha demostrado que un tratamiento térmico permite eliminar el flúor retenido en el interior de los nanotubos, incrementando la energía superficial y reduciendo el ángulo de contacto de las superficies con morfología nanotubular.

\section{DISCUSIÓN}

Es de gran interés, para el campo de los biomateriales, ajustar la mojabilidad de la superficie de los recubrimientos de $\mathrm{TiO}_{2}$ con morfología nanotubular, ya que esta propiedad puede tener influencia en la tasa de osteointegración de los implantes. Otras investigaciones previas se han centrado en el estudio de la mojabilidad y del crecimiento celular en la superficie de los nanotubos, sin pararse a explicar el fenómeno que produce que los nanotubos presenten un bajo ángulo de contacto.

Wang et al. (1998) indican que el ángulo de contacto del agua de las superficies de $\mathrm{TiO}_{2}$ varía tras haber sido almacenadas en la oscuridad o tras irradiarse con luz UV. Estas superficies de $\mathrm{TiO}_{2}$ presentaron un ángulo de contacto de $15^{\circ}$ tras haberse formado, después de haberse almacenado durante un periodo de 2 meses el ángulo de contacto se incrementó hasta los $72^{\circ}$, finalmente, tras iluminarse con luz UV se redujo hasta los $0^{\circ}$.

Hasta ahora, no se ha informado sobre el efecto que tiene el almacenaje en la oscuridad, así como un tratamiento térmico, en la mojabilidad, ángulo de contacto y energía superficial de los recubrimientos nanotubulares de óxido de titanio. En este trabajo se muestra que el almacenaje en la oscuridad de aleaciones de titanio con recubrimientos nanotubulares tiene efecto sobre la hidrofilidad de las superficies. Este tipo de recubrimiento con rugosidad a escala nanométrica incrementa el ángulo de contacto en agua a valores superiores a $130^{\circ}$, duplicando el obtenido por Wang et al. (1998). El anodizado electroquímico aumenta la energía superficial con respecto a las superficies desbastadas y grabadas con ácido y esto puede tener un impacto positivo en la biocompatibilidad de los implantes de titanio; ya que la componente polar de la energía superficial es superior en los recubrimientos nanotubulares que en las superficies desbastadas. Este fenómeno se explica principalmente por la mayor presencia de moléculas polares $\left(-\mathrm{OH}, \mathrm{TiO}_{2}\right.$, etc) introducidas por el proceso de anodizado electroquímico. Por otra parte, el menor valor del componente polar de las superficies grabadas con ácido se justifica por la reducción de la capa de óxido, producida por el proceso de limpieza al sumergirla en ácido nítrico 
tras el grabado ácido $(\mathrm{HF} / \mathrm{HCl})$. El aumento de la componente dispersiva de las superficies anodizadas se justifica con el incremento de la rugosidad a escala nanométrica, producida por la morfología nanotubular obtenida en el proceso de anodizado electroquímico. En definitiva, estas superficies con morfología y rugosidad a escala nanométrica permiten incrementar los contactos locales entre las células y la superficie del implante, esto juega un papel importante en la adhesión y proliferación de los osteoblastos, y por lo tanto el crecimiento del hueso (Das et al., 2008; Elias et al., 2008; Puckett et al., 2010a).

Los factores superficiales de rugosidad, energía superficial, composición química, fases presentes y resistencia a la corrosión todavía deben de estudiarse para mejorar la siguiente generación de implantes ortopédicos y entender las interacciones de las células con la superficie del implante. Para que se produzca la mineralización de la matriz extracelular, primero los osteoblastos deben de anclarse a la superficie del implante y posteriormente diferenciarse. Esta primera etapa de anclaje implica una unión físicoquímica entre las células y la superficie del biomaterial; el anclaje de las células se ve afectado por la morfología del implante, rugosidad superficial y composición química de la superficie. Basándose en los resultados recopilados de varios autores (Das et al., 2008; Puckett et al., 2010b; Sista et al., 2013; Chen et al., 2014) se puede predecir que el mayor grado de osteointegración y posterior biocompatibilidad se alcanzará cuando la aleación de titanio se someta a un proceso de anodizado electroquímico, seguido de un tratamiento térmico. Ambos procesos permiten eliminar el flúor retenido en los nanotubos, incrementando la energía y área superficial de contacto, así como modificar la estructura cristalina del óxido de titanio de amorfa a anatasa (Macak et al., 2007; Das et al., 2008). La formación de este recubrimiento de $\mathrm{TiO}_{2}$ con morfología nanotubular puede incrementar la tasa de osteointegración por medio el aumento de la superficie específica del implante y la menor tasa de liberación de iones. Así, la capa nanotubular de $\mathrm{TiO}_{2}$ puede interaccionar con el agua formando grupos Ti-OH y estos sirven como puntos de anclaje de los iones de calcio y favorecen la formación del hueso. Los grupos Ti-OH reaccionan con los iones de $\mathrm{Ca}^{2+}$, creando una carga positiva en la superficie, donde los grupos fosfato puede anclarse, formándose fosfato de calcio amorfo que incrementa la tasa de crecimiento óseo (Das et al., 2008).

Otra ventaja importante que presentan los nanotubos de óxido de titanio es que pueden ser empleados como depósitos de antibióticos o antibactericidas, los cuales pueden liberarse gradualmente en la interfase implante-tejido. Actualmente los tratamientos superficiales están diseñados para mejorar la tasa de osteointegración, o evitar la infección mediante esterilización, en lugar de prevenir la infección bacteriana en la interfase implantetejido. Estos tratamientos incluyen la modificación de la superficie mediante arenado, grabado ácido, combinación de ambos, o mediante la esterilización empleando rayos gamma. Los recubrimientos nanotubulares donde se incorporan factores de crecimiento, antibióticos e incluso nano partículas de plata permiten combatir la infección bacteriana en el foco de la misma e incrementar la tasa de osteointegración, mejorando el éxito clínico de los implantes (Chennell et al., 2013; Bayram et al., 2014; Çalışkan et al., 2014b; Chen et al., 2014). La cantidad de almacenamiento de estos compuestos depende de las dimensiones de los nanotubos que ajustarse en función de los parámetros del proceso de anodizado electroquímico.

Por lo tanto, es importante seguir investigando con el objetivo de conocer que parámetros de proceso de anodizado electroquímico y posteriores tratamientos (térmicos, deposiciones de compuestos, irradiación UV, etc.) son necesarios para alcanzar las propiedades óptimas (energía superficial, resistencia a la corrosión, topografía, rugosidad, liberación de antibióticos, etc.) del acabado superficial con el objetivo de incrementar la biocompatibilidad y ciclo de vida de los implantes.

\section{CONCLUSIONES}

- El proceso de anodizado electroquímico, en un electrolito compuesto por $\mathrm{H}_{3} \mathrm{PO}_{4}$ y NaF, modifica la topografía y la composición química de la superficie de la aleación de Ti6A14V ELI. El tratamiento superficial genera un recubrimiento de óxido de titanio con morfología nanotubular, presentando valores de ángulo de contacto, en agua, y energía superficial superiores a las superficies desbastadas o grabadas con ácido. Este fenómeno se explica principalmente por el fenómeno de Cassie-Bexter y por la ausencia de hidrofibicidad fotoinducida del óxido de titanio tras haberse almacenado en la oscuridad.

- El empleo de un tratamiento térmico, a una temperatura de $320^{\circ} \mathrm{C}$ y durante $30 \mathrm{~min}$, ha permitido reducir considerablemente el contenido de flúor retenido en los nanotubos, sin que se haya producido un colapso o derrumbe de éstos por el gradiente de temperatura, sin prácticamente alterar el ángulo de contacto ni la energía superficial.

\section{AGRADECIMIENTOS}

Al Ministerio de Economía y Competitividad del Gobierno de España por la financiación recibida a través del proyecto de investigación MAT201453764-C3-1-R. A la Generalitat Valenciana por el proyecto PROMETEO/2016/040. A la Comisión 
Europea por los fondos FEDER para la adquisición de equipamiento, y al servicio de Microscopía de la Universitat Politècnica de València, por su uso.

\section{REFERENCIAS}

ASTM F136 (2013). Standard Specification for Wrought Titanium-6Aluminum-4Vanadium ELI (Extra Low Interstitial) Alloy for Surgical Implant Applications (UNS R56401), ASTM International, West Conshohocken, PA, USA.

Bayram, C., Demirbilek, M., Yalçin, E., Bozkurt, M., Doğan, M., Denkbas, E.B. (2014). Osteoblast response on co-modified titanium surfaces via anodization and electrospinning. Appl. Surf. Sci. 288, 143-148. https://doi. org/10.1016/j.apsusc.2013.09.168

Berger, S., Hahn, R., Roy, P., Schmuki, P. (2010). Self-organized $\mathrm{TiO}_{2}$ nanotubes: Factors affecting their morphology and properties. Phys. Status Solidi B 247, 2424-2435. https:// doi.org/10.1002/pssb.201046373.

Bharathidasan, T., Narayanan, T.N., Sathyanaryanan, S., Sreejakumari, S.S. (2015). Above $170^{\circ}$ water contact angle and oleophobicity of fluorinated graphene oxide based transparent polymeric films. Carbon 84, 207-213. https://doi. org/10.1016/j.carbon.2014.12.004.

Çalışkan, N., Bayram, C., Erdal, E., Karahaliloğlu, Z., Baki, E. (2014a). Titania nanotubes with adjustable dimensions for drug reservoir sites and enhanced cell adhesion. Mat. Sci. Eng. C 35, 100-105. https://doi.org/10.1016/j. msec.2013.10.033.

Calișkan, N., Bayram, C., Erdal, E., Karahaliloğlu, Z., Denkbas, E.B. (2014b). Titania nanotubes with adjustable dimensions for drug reservoir sites and enhanced cell adhesion. Mat. Sci. Eng. C 35 (1), 100-105. https://doi.org/10.1016/j. msec.2013.10.033.

Chen, J., Zhang, Z., Ouyang, J., Chen, X., Xu, Z., Sun, X. (2014). Bioactivity and osteogenic cell response of $\mathrm{TiO}_{2}$ nanotubes coupled with nanoscale calcium phosphate via ultrasonification-assisted electrochemical deposition. Appl. Surf. Sci. 305, 24-32. https://doi.org/10.1016/j. apsusc. 2014.02.148.

Chennell, P., Feschet-Chassot, E., Devers, T., Awitor, K.O., Descamps, S., Sautou, V. (2013). In vitro evaluation of $\mathrm{TiO}_{2}$ nanotubes as cefuroxime carriers on orthopaedic implants for the prevention of periprosthetic joint infections. Int. J. Pharm. 455 (1-2), 298-305. https://doi.org/10.1016/j. ijpharm.2013.07.014.

Das, K., Bose, S., Bandyopadhyay, A. (2008). $\mathrm{TiO}_{2}$ nanotubes on Ti: Influence of nanoscale morphology on bone cellmaterials interaction. J. Biomed. Mater. Res. A 90 (1), 225237. https://doi.org/10.1002/jbm.a.32088.

Davis, J.R. (1990). ASM Handbook: Properties and Selection: Nonferrous Alloys and Special-Purpose Materials. Vol. 2, ASM International.

Duraccio, D. Mussano, F. Faga, M.G. (2015). Biomaterials for dental implants: current and future trends. J. Mater. Sci. 50 (14), 4779-4812. https://doi.org/10.1007/ s10853-015-9056-3.

Elias, C.N., Oshida, Y., Cavalcanti, J.H., Muller, C.A. (2008). Relationship between surface properties (roughness, wettability and morphology) of titanium and dental implant removal torque. J. Mech. Behav. Biomed. Mater. 1 (3), 234 242. https://doi.org/10.1016/j.jmbbm.2007.12.002.

Fais, L.M.G., Fernandes-Filho, R.B., Pereira-Da-Silva, M.A., Vaz, L.G., Adabo, G.L. (2012). Titanium surface topography after brushing with fluoride and fluoride-free toothpaste simulating 10 years of use. J. Dent. 40 (4), 265-275. https://doi.org/10.1016/j.jdent.2012.01.001.

Giacomello, A., Meloni, S., Chinappi, M., Casciola, C.M. (2012). Cassie-baxter and wenzel states on a nanostructured surface: Phase diagram, metastabilities, and transition mechanism by atomistic free energy calculations. Langmuir 28 (29), 10764-10772. https://doi.org/10.1021/ la3018453.
Herrero-Climent, M., Lázaro, P., Vicente Rios, J., Lluch, S., Marqués, M., Guillem-Martí, J., Gil, F.J. (2013). Influence of acid-etching after grit-blasted on osseointegration of titanium dental implants: In vitro and in vivo studies. J. Mater. Sci.-Mater. M. 24 (8), 2047-2055. https://doi. org/10.1007/s10856-013-4935-0.

Jeong, Y.H., Kim, W.G., Choe, H.C., Brantley, W.A. (2014). Control of nanotube shape and morphology on Ti-Nb(Ta)$\mathrm{Zr}$ alloys by varying anodizing potential. Thin Solid Films 572, 105-112. https://doi.org/10.1016/j.tsf.2014.09.057.

Kim, E.S. Jeong, Y.H., Choe, H.C., Brantley, W.A. (2013). Formation of titanium dioxide nanotubes on Ti-30Nb-xTa alloys by anodizing. Thin Solid Films 549, 141-146. https:// doi.org/10.1016/j.tsf.2013.08.058.

Kubota, S., Johkura, K., Asanuma, K., Okouchi, Y., Ogiwara, N., Sasaki, K., Kasuga, T. (2004). Titanium oxide nanotubes for bone regeneration. J. Mater. Sci. Mater. Med. 15 (9), 1031-1035. https://doi. org/10.1023/B:JMSM.0000042689.78768.77.

Lario-Femenía, J., Amigó-Mata, A., Vicente-Escuder, A., SegoviaLópez, F., Amigó-Borrás, V. (2016a). Desarrollo de las aleaciones de titanio y tratamientos superficiales para incrementar la vida útil de los implantes. Rev. Metal. 52 (4), e 084. https://doi.org/10.3989/revmetalm.084

Lario, J., Vicente, A., Amigó, A., Segovia, F., Amigó, V. (2016b). Influencia del voltaje en la formación de nanotubos en aleaciones $\alpha, \alpha+\beta$ y $\beta$ de titanio. XXXIV Congreso Anual de la Sociedad Española de Ingeniería Biomédica, Valencia, pp. $164-167$

Lario, J., Amigó, A., Segovia, F., Amigó, V. (2017). Formación de nanotubos de $\mathrm{TiO}_{2}$ mediante anodizado electroquímico en las aleaciones de colada y pulvimetalúrgicas de titanio. VI Congreso Nacional de Pulvimetalurgia y I Congreso Iberoamericano de Pulvimetalurgia, Ciudad Real, España.

Lee, K., Jeong, Y.H., Brantley, W.A., Choe, H.C. (2013). Surface characteristics of hydroxyapatite films deposited on anodized titanium by an electrochemical method. Thin Solid Films 546, 185-188. https://doi.org/10.1016/j. tsf.2013.04.077

Macak, J.M., Taveira, L.V., Tsuchiya, H., Sirotna, K., Macak, J., Schmuki, P. (2006). Influence of different fluoride containing electrolytes on the formation of self-organized titania nanotubes by Ti anodization. J. Electroceram. 16 (1), 29-34. https://doi.org/10.1007/s10832-006-3904-0.

Macak, J.M., Tsuchiya, H., Ghicov, A., Yasuda, K., Hahn, R., Bauer, S., Schmuki, P. (2007). TiO nanotubes: Selforganized electrochemical formation, properties and applications. Curr. Opin. Solid S. Mater. Sci. 11 (1-2), 3-18. https://doi.org/10.1016/j.cossms.2007.08.004.

Minagar, S., Wang, J., Berndt, C.C., Ivanova, E.P., Wen, C. (2013). Cell response of anodized nanotubes on titanium and titanium alloys. J. Biomed. Mater. Res. A. 101 (9) 2726-2739. https://doi.org/10.1002/jbm.a.34575.

Niinomi, M. (2008). Mechanical biocompatibilities of titanium alloys for biomedical applications. J. Mech. Behav. Biomed. Mater. 1 (1), 30-42. https://doi.org/10.1016/j. jmbbm.2007.07.001.

Owens, D.K., Wendt, R.C. (1969). Estimation of surface free energy of polymers. J. Appl. Polym. Sci. 13, 1741-1747. https://onlinelibrary.wiley.com/doi/pdf/10.1002/app. 1969.070130815.

Reclaru, L., Meyer, J.-M. (1998). Effects of fluorides on titanium and other dental alloys in dentistry. Biomaterials 19 (1-3), 85-92. https://doi.org/10.1016/S0142-9612(97)00179-8.

Roguska, A., Pisarek, M., Belcarz, A., Marcon, L., Holdynski, M., Andrzejczuk, M., Janik-Czachor, M. (2016). Improvement of the bio-functional properties of $\mathrm{TiO}_{2}$ nanotubes. Appl. Surf. Sci. 388, 775-785. https://doi.org/10.1016/j. apsusc.2016.03.128.

Puckett, S.D., Taylor, E., Raimondo, T., Webster, T.J. (2010a). The relationship between the nanostructure of titanium surfaces and bacterial attachment. Biomaterials 31 (4), 706713. https://doi.org/10.1016/j.biomaterials.2009.09.081.

Puckett, S.D., Lee, P.P., Ciombor, D.M., Aaron, R.K., Webster, T.J. (2010b). Nanotextured titanium surfaces for enhancing skin growth on transcutaneous osseointegrated devices. 
Acta Biomater. 6 (6), 2352-2362. https://doi.org/10.1016/j. actbio.2009.12.016.

Pypen, C.M.J.M., Plenk, H., Ebel, M.F., Svagera, R. Wernisch, J. (1997). Characterization of microblasted and reactive ion etched surfaces on the commercially pure metals niobium, tantalum and titanium. J. Mater. Sci. Mater. M. 8 (12), 781-784. https://doi. org/10.1023/A:1018568830442.

Sista, S. Nouri, A. Li, Y., Wen, C., Hodgson, P.D., Pande, G. (2013). Cell biological responses of osteoblasts on anodized nanotubular surface of a titanium-zirconium alloy. J. Biomed. Mater. Res. A 101 (12), 3416-3430. https://doi. org/10.1002/jbm.a.34638.

Szmukler-Moncler, S., Salama, H., Reingewirtz, Y., Dubruille, J.H. (1998). Timing of loading and effect of micromotion on bone-dental implant interface : Review of Experimental Literature. J. Biomed. Mater. Res. 43 (2), 192-203. https:// doi.org/10.1002/(SICI) 1097-4636(199822)43:2<192::AIDJBM14>3.0.CO;2-K

Toniollo, M.B., Galo, R., Macedo, A.P., Rodrigues, R.C.S. Ribeiro, R.F., Chiarello de Mattos, M.da G. (2012). Effect of fluoride sodium mouthwash solutions on cpTi: Evaluation of physicochemical properties. Braz. Dent. J. 23 (5), 496501. https://doi.org/10.1590/S0103-64402012000500005.

Toumelin-Chemla, F., Rouelle, F., Burdairon, G. (1996). Corrosive properties of fluoride-containing odontologic gels against titanium. J. Dent. 24 (1-2), 109-115. https:// doi.org/10.1016/0300-5712(95)00033-X

Truong, V.K., Lapovok, R., Estrin, Y.S., Rundell, S., Wang, J.Y., Fluke, C.J., Crawford, R.J., Ivanova, E.P. (2010). The influence of nano-scale surface roughness on bacterial adhesion to ultrafine-grained titanium. Biomaterials 31 (13), 3674 3683. https://doi.org/10.1016/j.biomaterials.2010.01.071.

Wang, R., Hashimoto, K., Fujishima, A., Chikuni, M., Kojima, E. Kitamura, A., Shimohigoshi, M., Watanabe, T. (1997). Light-induced amphiphilic surfaces. Nature 388, 431-432. https://doi.org/10.1038/41233.

Wang, R., Hashimoto, K., Fujishima, A., Chikuni, M., Kojima, E., Kitamura, A., Shimohigoshi, M., Watanabe, T. (1998). Photogeneration of Highly Amphiphilic $\mathrm{TiO}_{2}$ Surfaces. Adv. Mater. 10 (2), 135-138. https://doi.org/10.1002/(Sici)15214095(199801)10:2<135::Aid-Adma135>3.3.Co;2-D.

Zha, J., Ali, S.S., Peyroux, J., Batisse, N., Claves, D., Dubois, M., Kharitonov, A.P., Monier, G., Darmanin, T., Guittard, F., Alekseiko, L.N. (2017). Superhydrophobicity of polymer films via fluorine atoms covalent attachment and surface nano-texturing. J. Fluorine Chem. 200, 123-132. https:// doi.org/10.1016/j.jfluchem.2017.06.011.

Zhao, Y., Xiong, T., Huang, W. (2010). Effect of heat treatment on bioactivity of anodic titania films. Appl. Surf. Sci. 256 (10), 3073-3076. https://doi.org/10.1016/j. apsusc.2009.11.075. 